\title{
Tracking Performance of the Scintillating Fiber Detector in the K2K Experiment
}

\author{
B. J. Kim ${ }^{\mathrm{m}, *}$, T. Iwashita ${ }^{\mathrm{f}}$, T. Ishida ${ }^{\mathrm{c}}$, E. J. Jeon ${ }^{\mathrm{c}}$, \\ H. Yokoyama ${ }^{\ell}$, S. Aoki ${ }^{\mathrm{f}}$, H. G. Berns ${ }^{\mathrm{n}}$, H. C. Bhang ${ }^{\mathrm{m}}$,

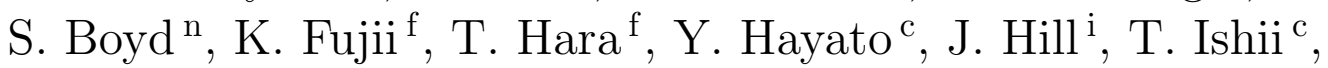 \\ H. Ishino ${ }^{\text {c }}$, C. K. Jung ${ }^{\text {i }}$, E. Kearns ${ }^{\text {a }}$, H. I. Kim ${ }^{\mathrm{m}}$, J. H. Kim ${ }^{\mathrm{m}}$, \\ J. Y. Kim ${ }^{\text {b }, ~ S . ~ B . ~ K i m ~}{ }^{\mathrm{m}}$, T. Kobayashi ${ }^{\mathrm{c}}$, S. Matsuno ${ }^{\mathrm{e}}$, \\ S. Mine ${ }^{d}$, K. Nakamura ${ }^{c}$, M. Nakamura ${ }^{\text {h }}$, K. Nishikawa ${ }^{g}$, \\ T. Otaki ${ }^{\text {f }}$, Y. Oyama ${ }^{\text {c }}$ H. Park ${ }^{\mathrm{c}, 1}$, M. Sakuda ${ }^{\mathrm{c}}$, K. Scholberg ${ }^{\mathrm{a}}$, \\ E. Sharkey ${ }^{i}$, J. L. Stone ${ }^{\text {a }}$, A. Suzuki ${ }^{f}$, K. Takenaka ${ }^{f}$, \\ N. Tamura ${ }^{\text {h }}$, Y. Tanaka ${ }^{\mathrm{f}}$, C. W. Walter ${ }^{\mathrm{a}}$, J. Wilkes ${ }^{\mathrm{n}}$, J. Yoo $^{\mathrm{m}}$, \\ and M. Yoshida ${ }^{\mathrm{k}}$ \\ ${ }^{a}$ Department of Physics, Boston University, Boston, MA 02215, USA \\ ${ }^{\mathrm{b}}$ Department of Physics, Chonnam National University, Kwangju 500-757, Korea \\ ${ }^{\mathrm{c}}$ High Energy Accelerator Research Organization, Tsukuba, Ibaraki 305-0801, \\ Japan \\ ${ }^{\mathrm{d}}$ Department of Physics and Astronomy, University of California, Irvine, CA \\ 92697-4575, USA \\ e Department of Physics and Astronomy, University of Hawaii, Honolulu, HI \\ 96822, USA \\ ${ }^{\mathrm{f}}$ Kobe University, Kobe, Hyogo 657-8501, Japan \\ ${ }^{\mathrm{g}}$ Department of Physics, Kyoto University, Kyoto 606-8502, Japan \\ ${ }^{\mathrm{h}}$ Department of Physics, Niigata University, Niigata, Niigata 950-2181, Japan \\ ${ }^{i}$ Department of Physics and Astronomy, State University of New York, Stony \\ Brook, NY 11794-3800, USA \\ jDepartment of Physics, Okayama University, Okayama, Okayama 700-8530, \\ Japan \\ ${ }^{\mathrm{k}}$ Department of Physics, Osaka University, Toyonaka, Osaka 560-0043, Japan \\ ${ }^{\ell}$ Department of Physics, Science University of Tokyo, Noda, Chiba 278-0022, \\ Japan \\ ${ }^{m}$ Department of Physics, Seoul National University, Seoul 151-742, Korea \\ ${ }^{\mathrm{n}}$ Department of Physics, University of Washington, Seatle, WA 98195-1560, USA
}




\begin{abstract}
The K2K long-baseline neutrino oscillation experiment uses a Scintillating Fiber Detector $(\mathrm{SciFi})$ to reconstruct charged particles produced in neutrino interactions in the near detector. We describe the track reconstruction algorithm and the performance of the SciFi after three years of operation.
\end{abstract}

Key words: Tracking, Scintillating, Fiber, Neutrino, Long-baseline PACS: 14.60.Pq, 29.40.Gx, 29.40.Mc

\title{
1 K2K Experiment
}

The $\mathrm{K} 2 \mathrm{~K}$ experiment is the first long-baseline neutrino oscillation experiment in operation. Muon neutrinos $\left(\nu_{\mu}\right)$ are produced via the decay of secondary pions from interactions of the KEK $12-\mathrm{GeV}$ proton beam in an aluminum target. The neutrino beam is directed through a near detector system at KEK to the Super-Kamiokande underground detector[1] 250km away. By comparing the neutrino fluxes and energy spectra measured at the near detector and at Super-Kamiokande, we are able to investigate neutrino oscillations. Details of the $\mathrm{K} 2 \mathrm{~K}$ experiment have been presented elsewhere [2].

The near detector, located $300 \mathrm{~m}$ downstream of the pion production point, consists of a $1 \mathrm{kt}$ water Cherenkov detector, a scintillating fiber tracking detector (SciFi), a hodoscope of plastic scintillation counters (VETO) around the SciFi, a lead glass detector (LG), and a muon range detector (MRD) [3]. It is illustrated in Fig. 1. The main purpose of the near detector is to measure the profile, flux, and energy spectrum of the neutrino beam. Among the near detector components, the SciFi can provide tracking capability good enough to discriminate neutrino interaction types and to measure energy and cross sections of the muon neutrino.

The K2K experiment started taking data in April, 1999. This paper will describe the performance of the Scintillating Fiber Detector after three years of operation. A description of the $\mathrm{K} 2 \mathrm{~K}$ scintillating fiber detector is given in Section 2. Hit reconstruction algorithm and performance are described in Section 3. Track and vertex reconstruction is described in Section 4. Performance

\footnotetext{
* Corresponding author. Tel.: +82-2-872-6868; fax: +82-2-884-3002.

E-mail address: bockjoo@neutrino.kek.jp (B. J. Kim).

1 Present address: Department of Physics, Seoul National University, Seoul 151-742, KOREA
} 


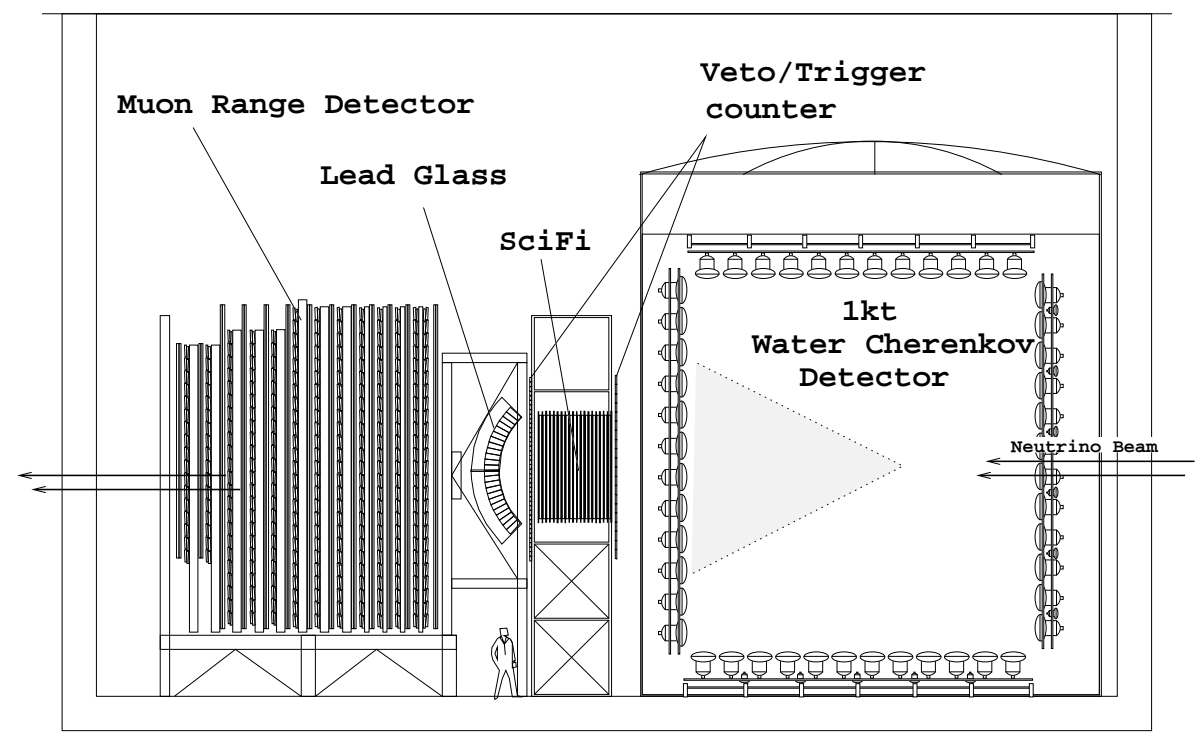

Fig. 1. The layout of the K2K near detector. It consists of a 1-kt water Cherenkov detector, a scintillating fiber detector, a hodoscope of plastic scintillation counters, a lead glass detector, and a muon range detector.

of the neutrino event reconstruction algorithms are presented in Section 5. We conclude in Section 6.

\section{Scintillating Fiber Detector}

The SciFi is a charged particle tracking detector using scintillating fiber technology. Scintillating fiber detectors have been used for charged particle trackers by other experiments [4]. A scintillating fiber detector was chosen for this application since it was necessary to have high tracking efficiency for reconstructing charged particles and their vertex positions when more than two visible particles are produced in neutrino interactions. Using the detector, we can separate quasi-elastic events from inelastic events, and define the fiducial volume of the neutrino interactions with $1 \%$ accuracy.

The water is contained in a thin aluminium structure, and the water layers are interleaved with scintillating fiber layers. Water is chosen as a target material in order to minimize systematic uncertainties in neutrino cross-section when the event rates are compared between the SciFi and Super-Kamiokande detectors. A scintillating fiber layer provides the hit-position measurement in both horizontal and vertical directions ${ }^{2}$. An individual scintillation sheet has

$\overline{2}$ In the K2K coordinate system, the $z$-axis is along the nominal neutrino beam direction. $y$-axis is the vertical direction pointing upwards, and $x$-axis is the horizontal direction. 


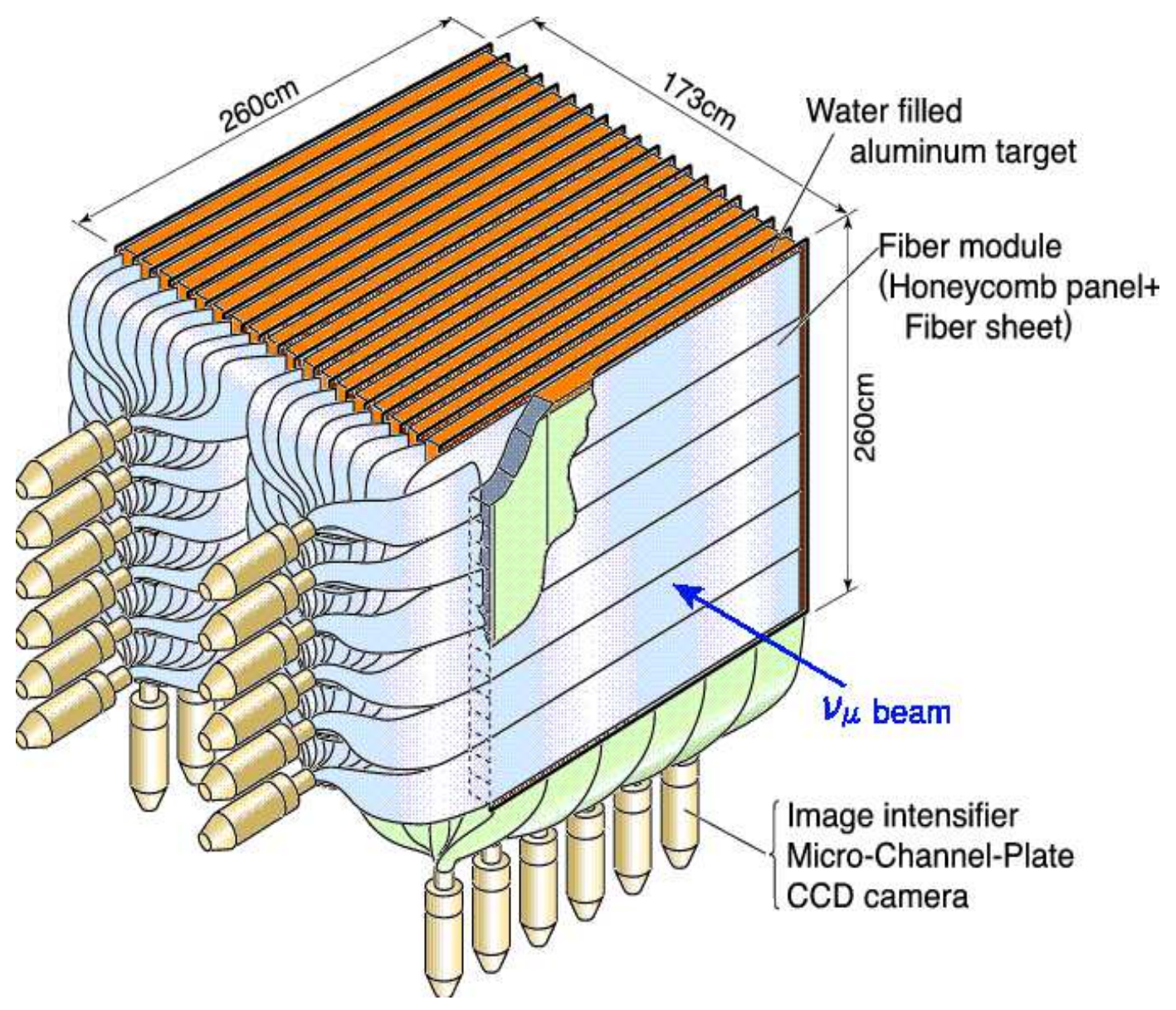

Fig. 2. A schematic view of the K2K scintillating fiber detector. The detector has the dimension of $260 \mathrm{~cm} \times 260 \mathrm{~cm} \times 173 \mathrm{~cm}$ in $(x, y, z)$. The central fiducial mass region is defined to be $220 \mathrm{~cm} \times 220 \mathrm{~cm} \times 159 \mathrm{~cm}$ in $(x, y, z)$.

a thickness of $1.3 \mathrm{~mm}$, consisting of two staggered layers of scintillating fibers. Each fiber has a diameter of $0.7 \mathrm{~mm}$. The vertical and horizonal fiber sheets are separated by a $1.6-\mathrm{cm}$ thick honeycomb panel. There are 20 scintillating fiber layers in total, and the adjacent layers are spaced $9 \mathrm{~cm}$ apart along the neutrino beam direction.

One layer of $240 \mathrm{~cm} \times 240 \mathrm{~cm} \times 6 \mathrm{~cm}$ water target, consisting of 15 aluminium tanks, is interleaved with two fiber layers. The wall thickness of the aluminium tank is $1.8 \mathrm{~mm}$. The weights of water, aluminium, and the honeycomb panel are 6.0tons, 1.4tons, and 0.8tons, respectively. A schematic view of the SciFi is shown in Fig. 2.

For processing light signals, a group of fibers are bundled and coupled to an image intensifier tube (IIT), an optical lens, and a CCD camera as shown in Fig. 2. A total of 24 IIT's are used to read out all 274080 fibers in the SciFi. In order to find the correspondence between the hit fiber and the CCD images, a certain number of selected fibers are illuminated periodically at the 
(a)

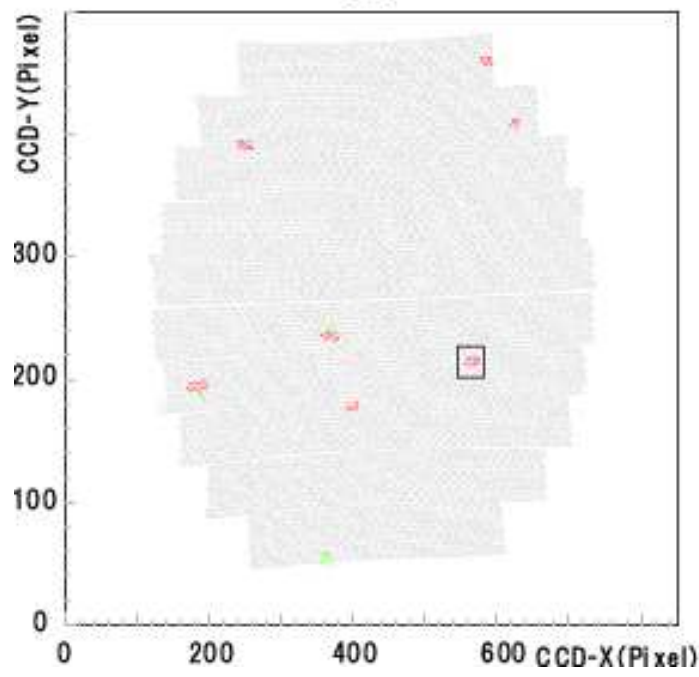

(b)

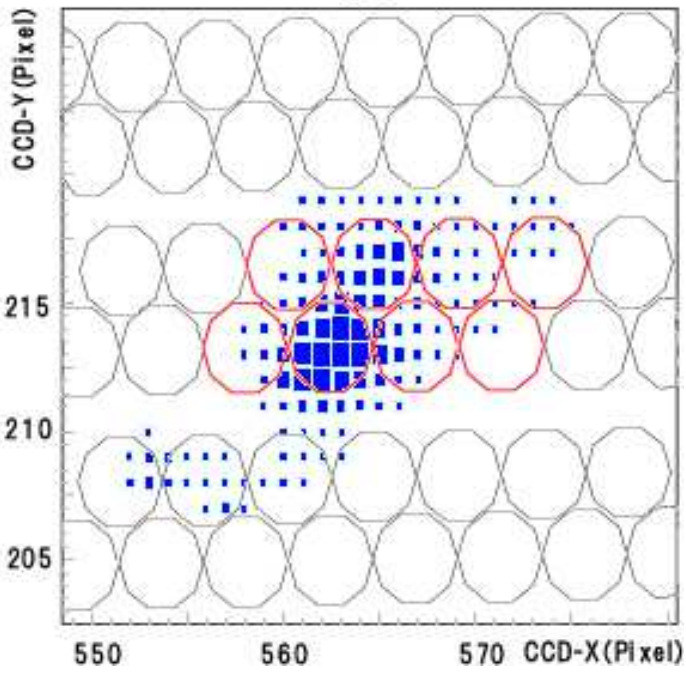

Fig. 3. (a) A typical CCD image of an IIT from a cosmic-ray event. Small distortions of the CCD image are introduced due to the position dependence of pixel size at the IIT-CCD contact. (b) An expanded view of (a) for a typical SciFi hit. The size of hit pixel is proportional to brightness in the ADC. Hit fibers are represented by the thick circles. A "SciFi hit" is defined as a cluster of neighboring hit fibers.

opposite side of IIT's by an electro-luminescent (EL) plate. This is referred to as the EL calibration. A more detailed description of the SciFi detector is found elsewhere [5].

\section{Hit reconstruction}

\subsection{Hit Finding Algorithm}

The CCD pixel images need to be handled to reconstruct hits in the SciFi. Fig. 3(a) shows a typical CCD image of an IIT from a cosmic-ray event. A fiber bundle, consisting of ten fiber sheets, is arranged to cover a sensitive region of an IIT effectively. Fig. 3(b) is an expanded view of (a), illustrating a typical "SciFi hit", when a cosmic-ray muon passes through the fiber sheet. The hit reconstruction is performed in the following four steps:

1. Loading Hit Pixels The raw data of the SciFi consist of hit pixels in the CCD coordinates $(x, y)$ and 8 bits of ADC brightness. These are all encoded in one byte per pixel. Typically, there are 3,500 hit pixels in a neutrino event.

2. Finding Pixel Clusters A pixel cluster is determined by a group of neighboring hit pixels. At this stage, isolated single pixel hits, coming from random electrical noise of $\mathrm{CCD}$, are rejected. 


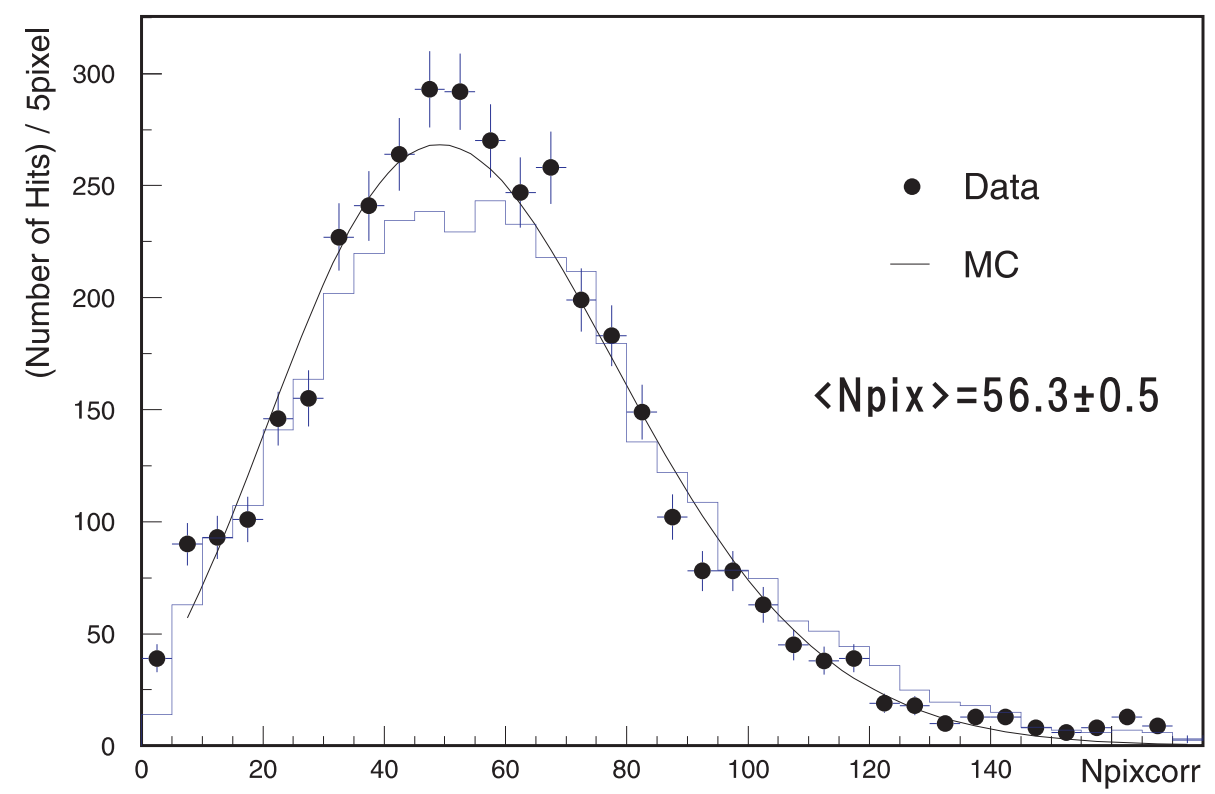

Fig. 4. An $N_{\text {pix }}$ distribution with the corrections applied for both attenuation and dependence on the incident angle as given in Eq.(1).

3. Identifying Hit Fibers A hit fiber is identified by requiring at least one or two hit pixels of cluster within the fiber. The hit fiber position in the CCD coordinate is determined from a mapping table that is obtained from the EL calibration.

4. Making Hit Fiber Clusters A fiber cluster is determined by a group of neighboring hit fibers.

We apply two kinds of filtering algorithms to the fiber clusters to improve the signal-to-noise ratio:

- We require that a fiber cluster should consist of hit fibers in both upper and lower layers of a fiber sheet. This eliminates fiber clusters of small pixel images which come from dark current in an IIT.

- When a big pixel cluster lies on the adjacent fiber sheets in a bundle and makes multiple fiber clusters, we choose only fiber clusters containing peak of ADC brightness. This removes fake fiber-clusters introduced by the dense arrangement of fiber sheets in a bundle.

We consider a fiber cluster passing through these two filters as a SciFi "hit". Every hit position in the CCD coordinate is then converted into the position in the detector coordinates $(x, z$ or $y, z)$. The hit position is defined as the centroid of a fiber cluster, weighted by the ADC value of the hit pixel. 


\subsection{Number of pixels per hit $\left(N_{\text {pix }}\right)$ and its corrections}

A found hit gives the number of pixels for the parent pixel cluster, $N_{\text {pix }}$. LED calibration data show that $N_{\text {pix }}$ has good linearity with respect to the number of photons injected to IIT. Since the intensity of observed light is proportional to the energy loss in the fiber, $N_{p i x}$ is expected to be a good estimator of the energy of the parent particle.

Fig. 4 shows an $N_{\text {pix }}$ distribution for cosmic-ray muons. Based on a reconstructed track, $N_{p i x}$ can be corrected to account for both the attenuation of light in the fiber, and the dependence of light yields on the incident angle $\left(\theta_{i n}\right)$ of a particle on a fiber sheet:

$$
N_{\text {pixcorr }}=\frac{F\left(l_{0}\right)}{F(l)} \times \cos \theta_{\text {in }} \times N_{\text {pix }} .
$$

The first term is the correction for attenuation of the scintillation light in the fiber and reflection at the far end of fiber coated with aluminum [5]. The correction is normalized by the value in case of the light from the detector center $\left(l_{0}=130 \mathrm{~cm}\right)$. Thus, $F(l)$ is given by:

$$
F(l)=\exp \left(-\frac{l+l_{g}}{\lambda}\right)+R \cdot \exp \left(-\frac{2 \cdot L-l+l_{g}}{\lambda}\right),
$$

where $l$ is the distance from the hit position to the near end of a fiber, $l_{g}(=100 \mathrm{~cm})$ is light guide length from the near end to the IIT, and $\lambda(=323 \mathrm{~cm})$ is the attenuation length. $R(=0.74)$ is the reflection coefficient at the far end of a fiber, and $L(=254 \mathrm{~cm})$ is the length of a fiber.

The second term in Eq. (1) is the correction for the dependence of light yields on the incident angle with respect to the fiber sheet. Here, $\theta_{i n}=0$ is perpendicular to the fiber sheet. $N_{\text {pixcorr }}$ is thus equivalent to $N_{\text {pix }}$ when a particle passes fiber sheet at the center of the detector $x / y$ planes with $\theta_{i n}=0$.

Figs. 5(a) and (b) show average values of $N_{\text {pix }}\left(\left\langle N_{\text {pix }}\right\rangle\right){ }^{3}$ as a function of hit position along the fiber and as a function of $1 / \cos \left(\theta_{i n}\right)$, respectively.

$\overline{3}$ We employ the following Poisson function to fit the $N_{p i x}$ distribution:

$$
F\left(N_{p i x} ; A, \alpha,\left\langle N_{p i x}\right\rangle\right)=A \cdot \frac{e^{-\mu} \times \mu^{x}}{\Gamma(x+1)}, \quad \mu \equiv\left\langle N_{p i x}\right\rangle / \alpha, \text { and } x \equiv N_{p i x} / \alpha,
$$

where $A$ is an overall normalization factor and $\alpha$ is a conversion factor. $\left\langle N_{p i x}\right\rangle$ is the average value of the $N_{p i x}$ distribution. 
(a)

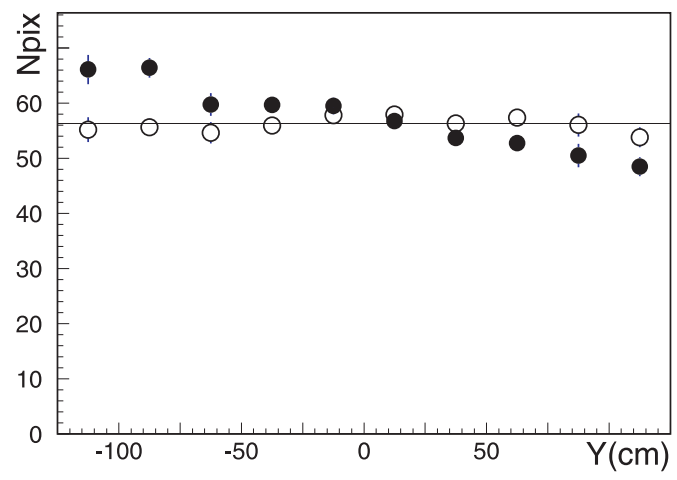

(b)

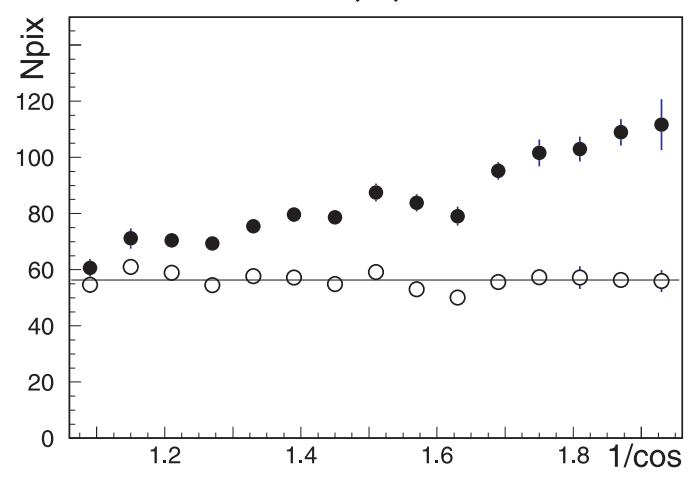

Fig. 5. An average value of $N_{p i x},\left\langle N_{\text {pix }}\right\rangle$, as a function of (a) hit position along the fiber and (b) $1 / \cos \theta_{\text {in }}$, where $\theta_{\text {in }}$ is the incident angle with respect to the fiber sheet, respectively. Solid (open) circles are before (after) the correction. The negative $x, y$ coordinates in (a) correspond to positions close to readout end of the fiber, and a larger value of $\left\langle N_{p i x}\right\rangle$ is expected before the correction.

\subsection{Simulation of CCD hit pixels}

To reproduce SciFi hits accurately in the K2K Monte Carlo simulation, we include simulation of hit pixels on CCD due to charged particles and noise in the following three steps:

1. Charged particles are tracked through the detector using GEANT[6]. The energy loss of a particle traversing a fiber is computed and then converted to the number of photoelectrons observed by the IIT/CCD imaging system, taking into account attentuation along the fiber, reflection by the aluminium coated at the end of a fiber, and the quantum efficiency of the IIT photocathodes.

2. The photoelectrons are converted to an image on the CCD camera surface. Individual photoelectrons are distributed around the fiber center according to a gaussian distribution with parameters determined using data of a single photoelectrons from LED calibration run. The size of the pixel cluster corresponding to the single photoelectron is then chosen from distributions of the LED data, and the pixel cluster is simulated accordingly. The energy scale is tuned for each IIT using cosmic-ray muon data.

3. Random noise in a pixel cluster is generated for each IIT based on neutrinobeam data.

Two kinds of noise pixels are generated. One is isolated single hit pixels around the main pixel cluster of a charged track in step 2.. The other is random noise pixels in a pixel cluster generated in step 3.. The total number of noise hits averaged over IITs is 1.67 for MC and 1.72 for cosmic-ray data. Based on the pixel simulation, the average number of noise hits is expected to be 70 per event. In the cosmic-ray data we find 52 hits per event due to the random 


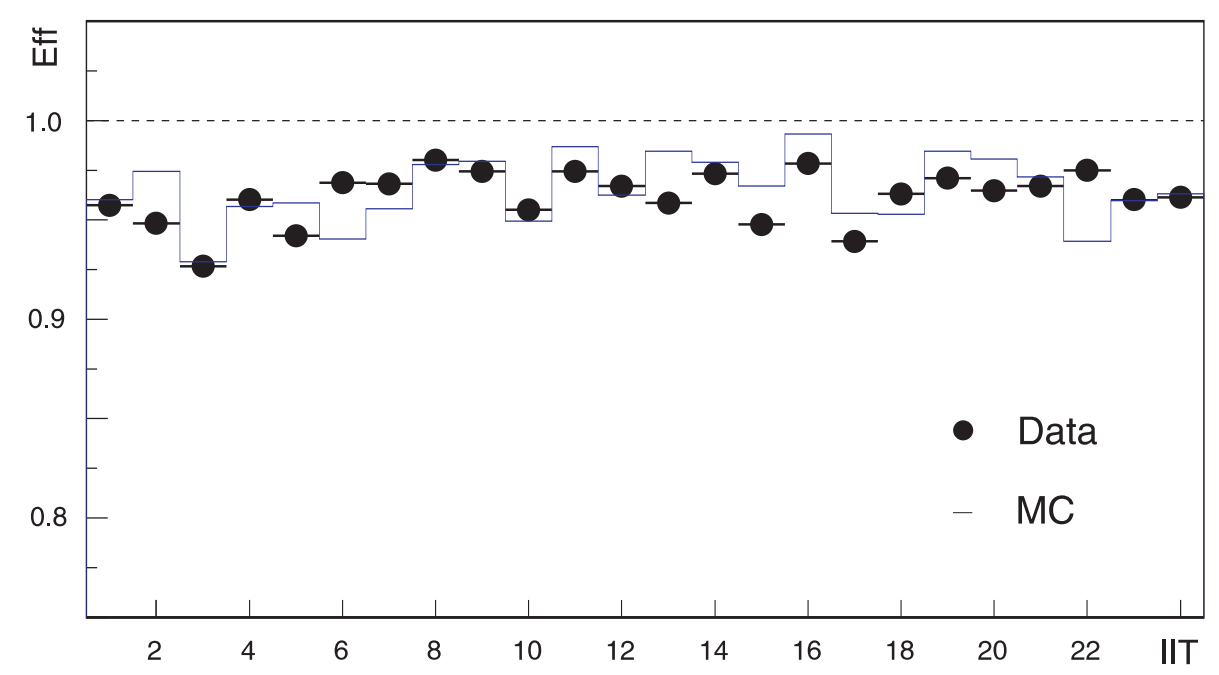

Fig. 6. Hit finding efficiency $\epsilon_{\text {hit }}$ obtained from cosmic-ray data

noise of CCD, and 17 hits per event due to the beam associated background. The noise rate is quite low, considering the total number of fibers $(274,080)$.

\subsection{Efficiency and Stability of Hit Reconstruction}

We can obtain the hit finding efficiency $\epsilon_{h i t}$ which is defined as:

$$
\epsilon_{\text {hit }}=\frac{\text { Number of observed hits }}{\text { Number of expected hits }}
$$

where the expected number of hits (denominator) is obtained from the information of reconstructed tracks. Fig. 6 shows $\epsilon_{\text {hit }}$ for each IIT. Data and Monte Carlo shows a good agreement.

Cosmic-ray data are taken during the normal data-taking period, roughly once every two weeks. The efficiency is monitored using the data as a check of detector stability.

We have performed a similar check on the stability of hit finding efficiency using the neutrino data. Clean single track events are selected by requiring a track with the number of hit layers larger than nine, and its matching to activity in the downstream VETO counter.

Fig. 7 shows hit finding efficiency, which is obtained by taking average over all IIT's, as a function of data-taking period. The dotted line at $96.8 \%$ represents an MC prediction. For the cosmic-ray data, we find an average value of $\epsilon_{\text {hit }}$ to be $96.5 \pm 0.5 \%$ for all IIT's and all time periods. 4 .

$\overline{4}$ This is in good agreement with a simple estimation. When a minimum ionizing 


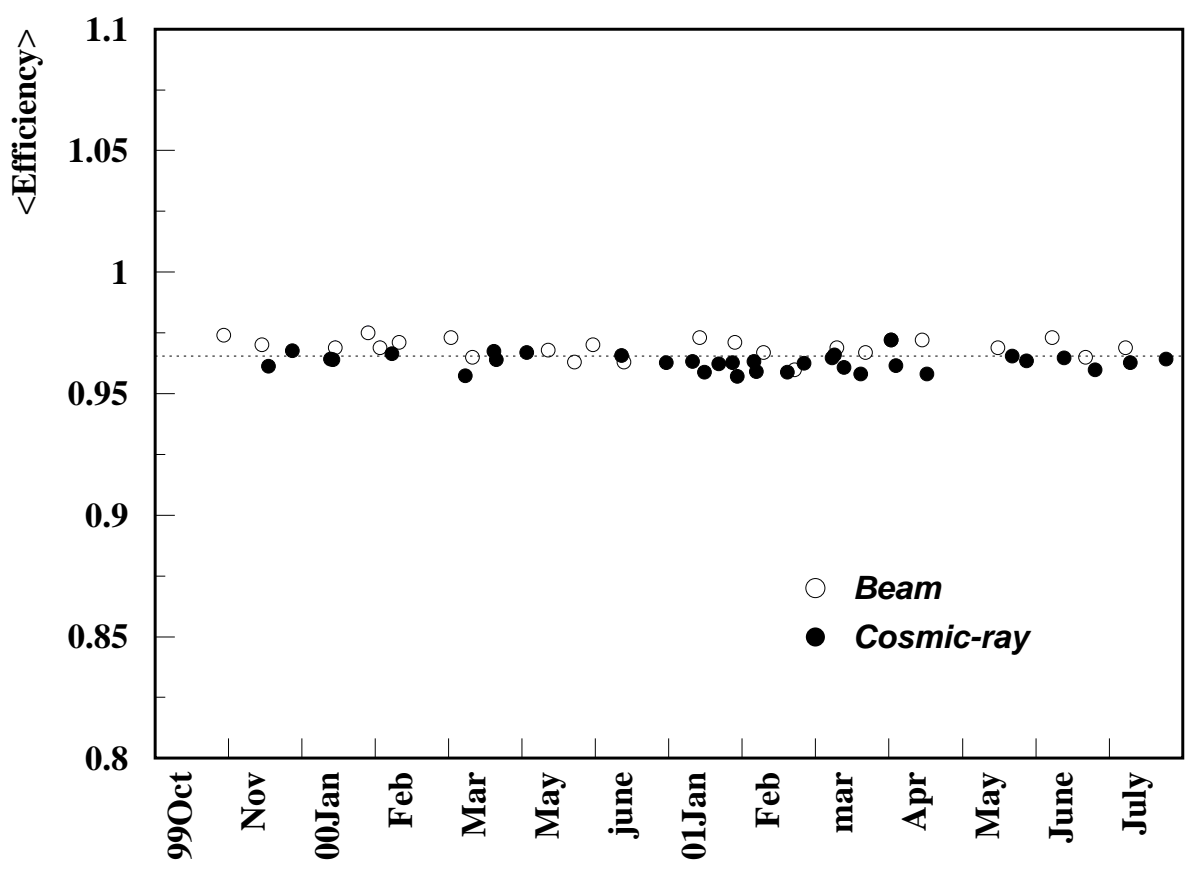

Fig. 7. Hit finding efficiency as a function of data taking period for the neutrino data (open circle) and cosmic ray data (solid circel). The dotted line represents an $\mathrm{MC}$ predicted hit finding efficiency.

\section{Reconstruction of Track and Vertex}

\subsection{Track Reconstruction Algorithm}

The track finding algorithm in the SciFi is optimized for the charged current interactions which contain a muon in the final state. The track finding algorithm consists of three steps.

First, the track finder searches for 2-dimensional (2D) track candidates in the $(x, z)$ or $(y, z)$ planes which traverse at least three layers of SciFi. A 3dimensional track is reconstructed by selecting the best combination of two $2 \mathrm{D}$ tracks. In order to reduce the combinatorial fake tracks, the starting and ending hit layers are required not to be different by more than one layer, and the overlapping of the two $2 \mathrm{D}$ tracks is required to be at least two layers.

Secondly, the combinatorial background or noise tracks are removed using other components of the near detector (VETO and MRD). A track candidate in the SciFi is required to match with both a hit in the VETO and a track in

particle crossing the fiber sheet creates $\sim 8 p . e$. (4p.e. each for upper and lower layers), hit coincidence probability between upper and lower layers is $\left(1 .-e^{-4}\right)^{2}=0.96$. The requirement of the coincidence between two fiber layers is chosen to keep the noise hits at the level of 70 hits per event. 
the MRD.

Once a good primary track is found, the most upstream hit-position of the primary track is considered as a neutrino interaction point. The track finder starts searching for extra tracks near the neutrino interaction point. These tracks are mostly short tracks with two or more SciFi hits. This method of extra track finding turns out to be an effective way to find short tracks of protons and/or pions from the neutrino interactions. All the reconstructed tracks are subjected to a "global fit", a straight line fit to the found hits.

\subsection{Alignment and Position Resolution}

The geometry of the SciFi and its support structure was surveyed by mechanical means after their installation was completed. Based on the mechanical survey, the alignment of fibers is estimated to be good to within $5 \mathrm{~mm}$.

A better alignment was obtained by minimizing the sum of the squared hitresiduals using the global fit for cosmic ray tracks. From the mean shift of the hit residuals, we find the accuracy of the alignment to be $\sim 400 \mu \mathrm{m}$.

Multiple scatterings occurring in the SciFi is not negligible compared with the size of a fiber. A Kalman filtering technique [7] [8] [9] can be employed to take the multiple scattering into account and, in principle, removes the multiple scattering effect during track fitting. The technique calculates the error of a track fit relatively quickly, and avoids the tedious matrix inversion by the global fit. A track fit using the Kalman filtering technique is used to estimate the detector resolution more accurately and to obtain a better alignment. The implementation adopts the prescriptions described in Refs. [9] and [10].

Some curvature was introduced in the fiber sheets due to bending when they were glued to a fiber module. Production of the fiber modules is described in Ref. [5]. The curvature of a fiber sheet causes displacement from the aligned fiber-position and results in a rotational misalignment. The bending of every fiber sheet was measured after each fiber module was assembled. The curvature of a fiber sheet was then obtained by fitting the measured fiber bendings to a forth-order polynomial, and used to correct the rotational misalignment.

After the Kalman filtering and the curvature correction, the accuracy of alignment becomes better than $50 \mu \mathrm{m}$, estimated from the mean residual shift. Fig. 8 shows distributions of the mean and standard deviation of the hit residuals for the mechanical survey alone, the alignment using the global fit, and the alignment using the Kalman filtering fit.

The mean of hit residual after the mechanical survey alone shows a variation 

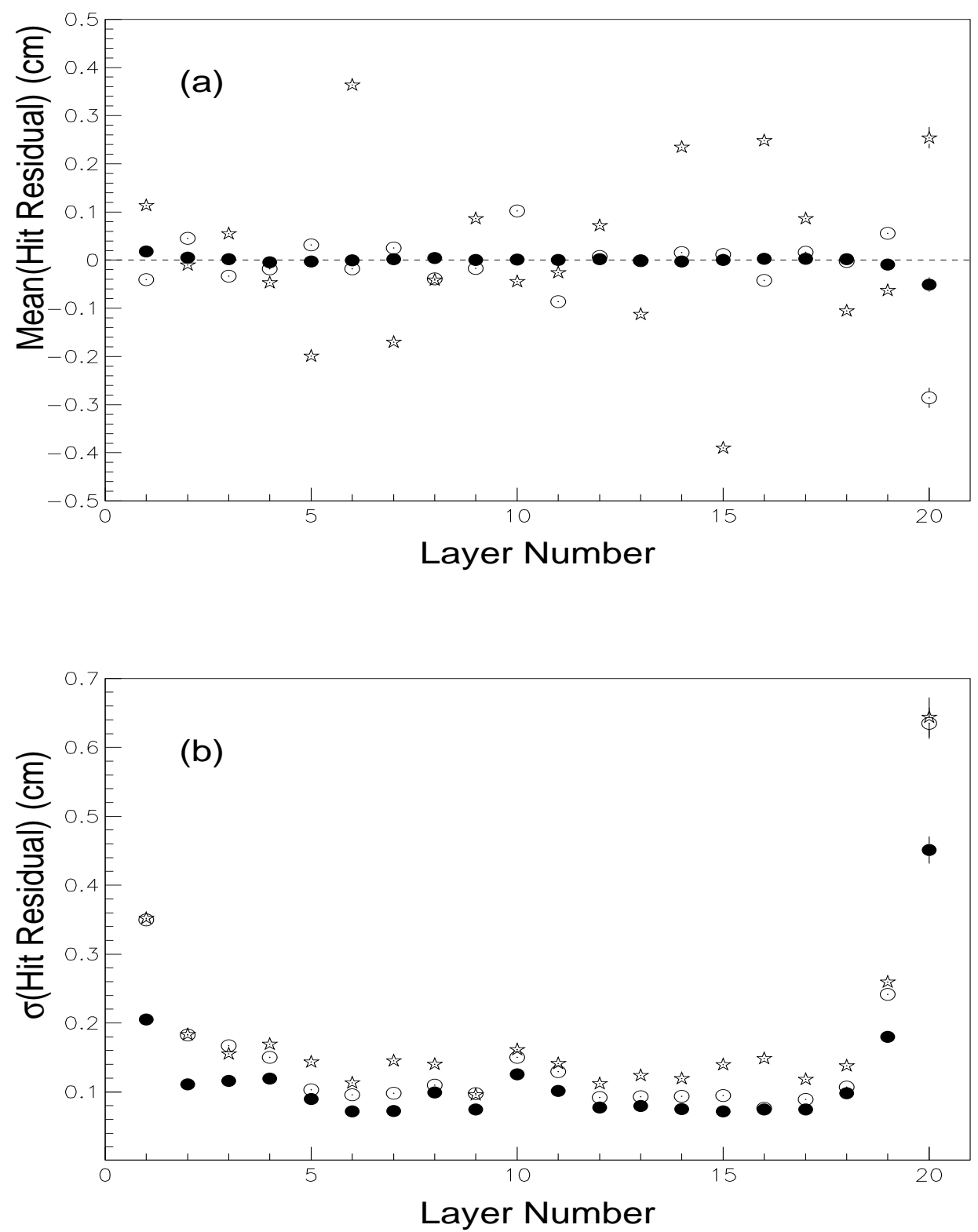

Fig. 8. (a) Means of hit residual and (b) standard deviations as a function of layers in $y-z$ plane. Points are for the mechanical survey alone (open star), the alignment using the global fit (open circle), and the alignment using the Kalman filtering (solid circle).

as large as $1 \mathrm{~cm}$. After the alignment using the global fit, the variation becomes smaller than $400 \mu \mathrm{m}$. After the alignment using the Kalman filtering fit, the variation becomes at most $50 \mu \mathrm{m}$. The standard deviations of the hit residual also exhibit such improvements of fiber alignment as the mean values. There are a few layers with large standard deviations of hit residual, compared with other layers. These are the outer most layers and indeed bent more than the 
rest of inner layers.

The alignment is checked using periodically taken cosmic-ray data. Fig. 9 shows the variation in the mean values of hit residuals since May 1999, and the variation is found to be less than $100 \mu \mathrm{m}$.

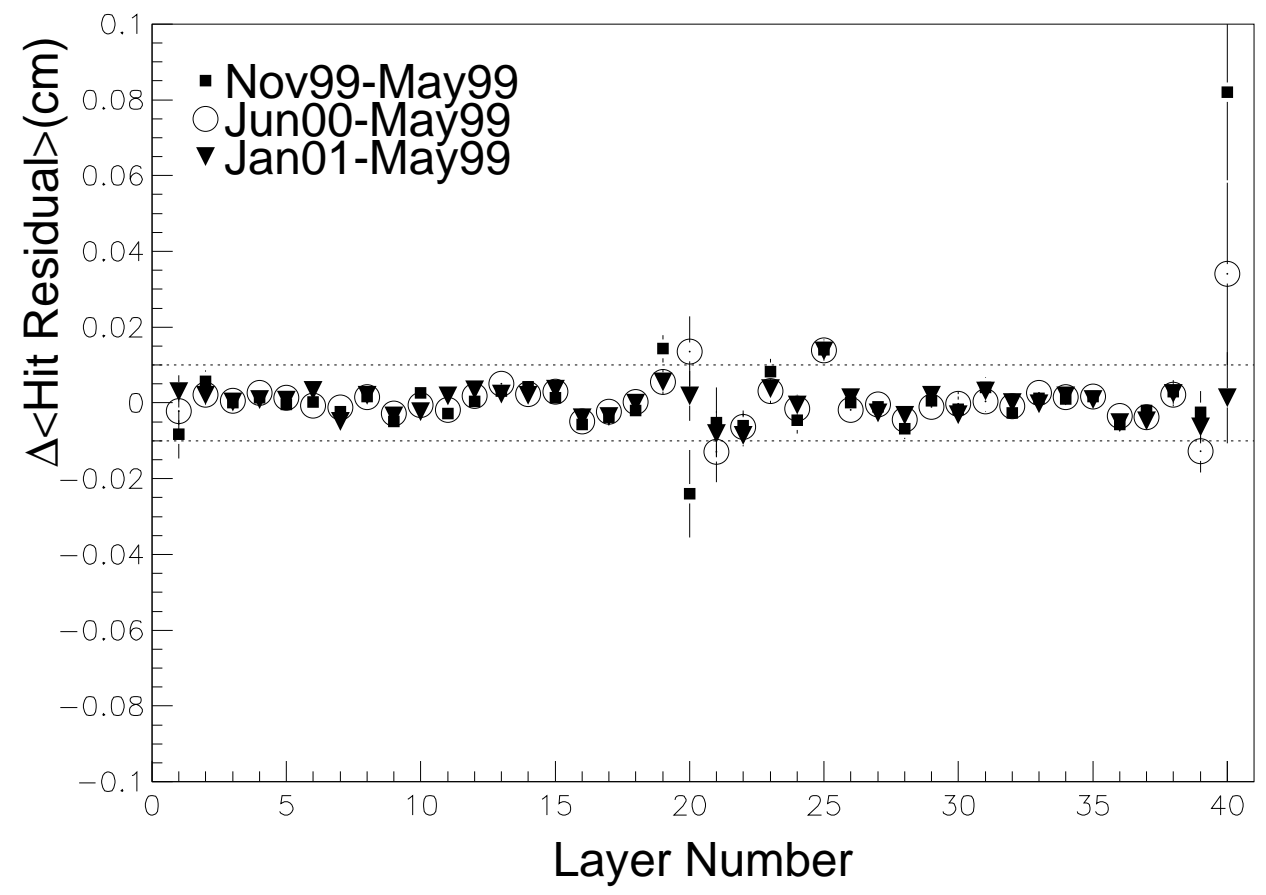

Fig. 9. The variation in the mean of hit residual, obtained from cosmic-ray data, as a function of layers in $x-z$ plane (1-20) and $y-z$ plane (21-40). The variation is taken relative to May 1999. The dotted line represents the variations of $\pm 100 \mu \mathrm{m}$.

The neutrino data with the Kalman filtering fit are used to obtain the best estimation for the position resolution. Fig. 10 shows the hit residual distribution using neutrino data. The position resolution is $0.73 \mathrm{~mm}(0.61 \mathrm{~mm})$ for muon energy $\mathrm{E}_{\mu}<1 \mathrm{GeV}\left(\mathrm{E}_{\mu}>1 \mathrm{GeV}\right)$. The overall position resolution is $0.64 \pm 0.07 \mathrm{~mm}$. Fig. 11 shows the energy dependence of the resolution. The effect of multiple scattering on the position resolution decreases as the muon energy increases. The MC prediction explains the overall energy dependence. The difference between data and $\mathrm{MC}, \sim 100 \mu \mathrm{mm}$, is regarded as the systematic error of our detector. The position resolution of $0.6 \mathrm{~mm}$ is regarded as that of the SciFi without the multiple scattering effect 

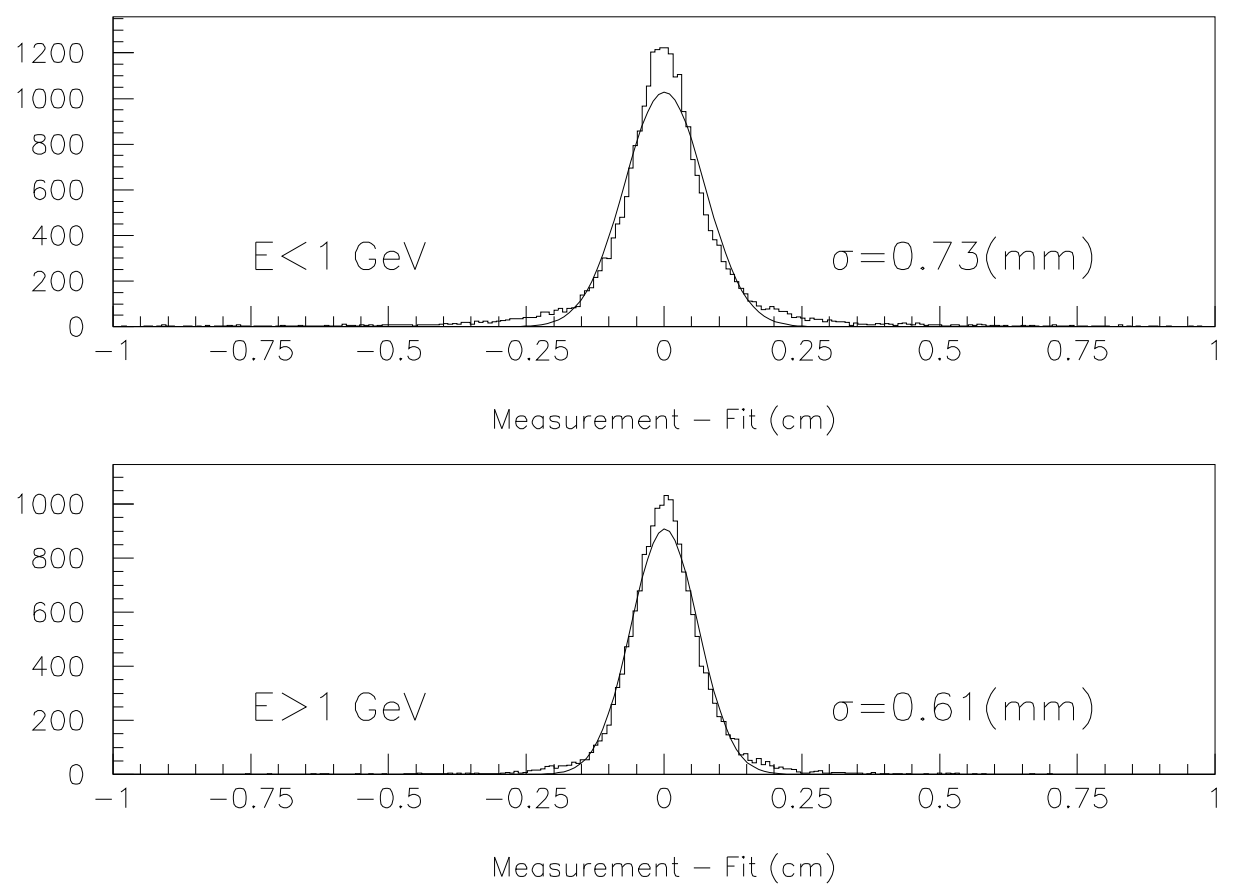

Fig. 10. The hit residual distributions using the neutrino beam data with the Kalman filtering fit. The curve is a single Gaussian fit to the data with $\sigma=0.73 \mathrm{~mm}(0.61 \mathrm{~mm})$ for muon energy $\mathrm{E}_{\mu}<1 \mathrm{GeV}\left(\mathrm{E}_{\mu}>1 \mathrm{GeV}\right)$.

\subsection{Track Finding Efficiency}

The efficiency for finding a single muon track is estimated from cosmic-ray and MC events. The cosmic-ray events are selected by requiring associated activities in both upstream and downstream VETO counters. Furthermore, tracks are required to have at least $15 \mathrm{SciFi}$ hit layers from the upstream to the downstream. In order to study dependence of the track finding efficiency on track length, we artificially eliminate hits in the upstream side of the track. For example, if it is desired to estimate the efficiency of the track with ten SciFi hit layers using 19-layer long track, the first to the nineth hit layers are eliminated before applying a track finding algorithm. For MC events, a muon track is required to have at least three hit-layers within the SciFi fiducial volume, and tested by a track finding algorithm.

Fig. 12 shows a comparison of single track finding efficiency as a function of the track length in unit of number of SciFi layers for the cosmic ray data. Track finding inefficiencies come from failures in the hit finding and imperfection of the track reconstruction. The track finding efficiency is $\sim 70 \%(\sim 87 \%)$ for the track length of three (four) hit SciFi layers and becomes close to $100 \%$ for 


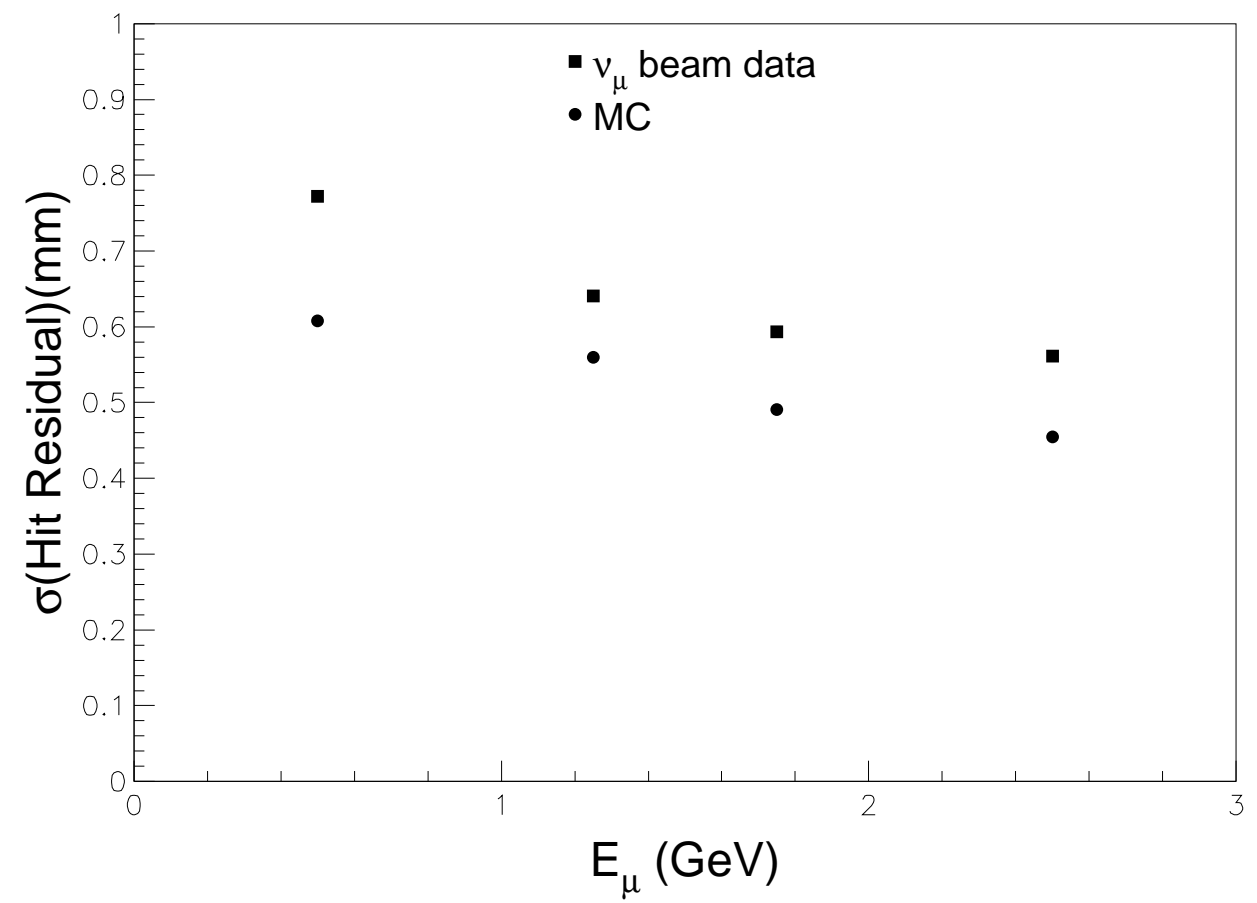

Fig. 11. The energy dependence of position resolution.

longer tracks.

An average track finding efficiency for the cosmic ray events is estimated by weighting the track length distribution obtained from the MC. We find the average track finding efficiency to be $93.6 \pm 0.8 \%$ for cosmic-ray tracks with three hits or more. The average track finding efficiency is estimated to be $92.7 \pm 0.5 \%$ for the MC events.

\subsection{Vertex Reconstruction}

An accurate fiducial volume is crucial for measuring the cross section of neutrino in the SciFi. Therefore, the event vertex has to be determined precisely.

The vertex position is determined by the reconstructed tracks. The event vertex for a single track event is found by simply taking the mid-point of the upstream water container nearest to the first hit in the $z$-direction of the reconstructed track. The event vertex for a multiple track event is determined by taking average of the $z$ positions at intersections of a primary track and the rest of shorter tracks. Fig. 13 shows a comparison of $x$ - and $z$-vertex distributions between data and MC for both single and multiple track events. 


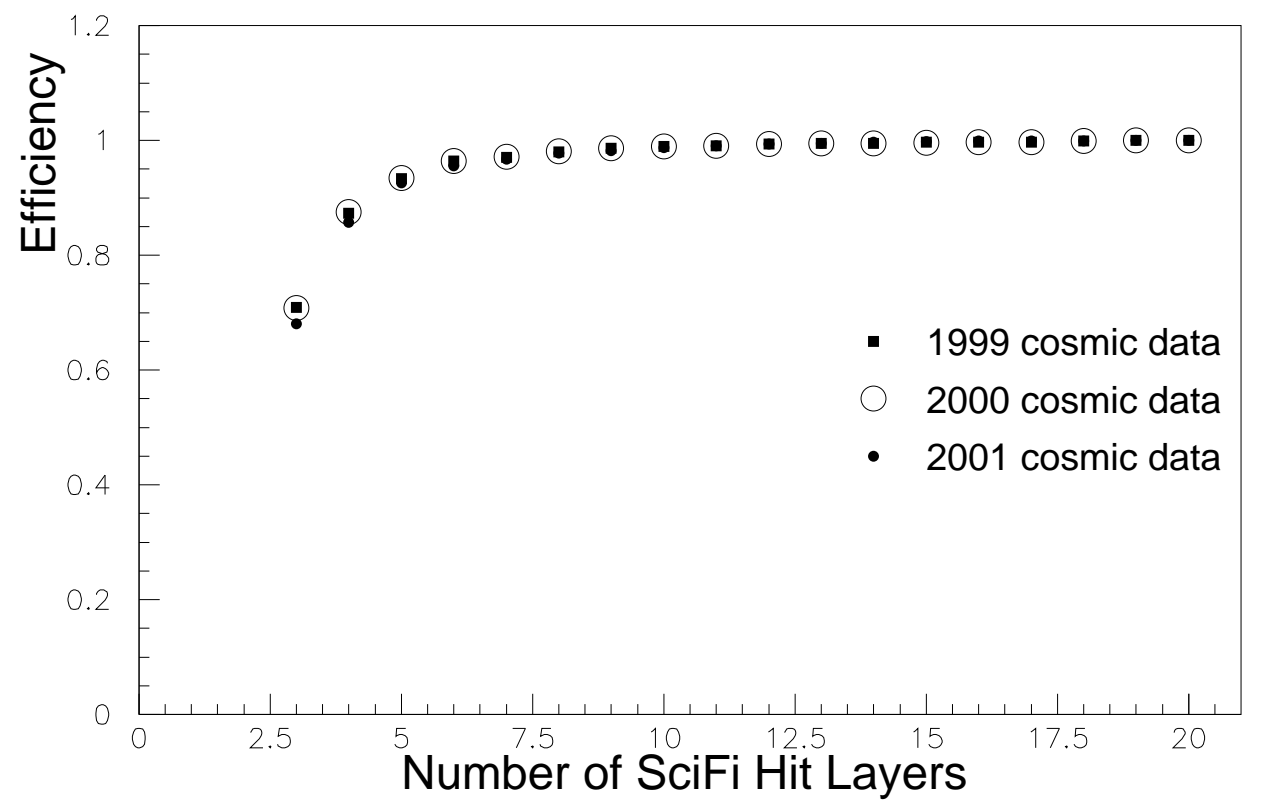

Fig. 12. The primary track finding efficiency as a function of track length using cosmic ray data. Solid rectangle, open circle, and solid circle are for 1999, 2000, and 2001 cosmic ray data, respectively. The track length is given in units of number of SciFi layers.

Events with tracks ranged out at the MRD are used for the comparison and the comparison shows a good agreement between data and MC.

For two track events, $z$-vertex difference between $(x, z)$ and $(y, z)$ planes can provide a measure of goodness for the vertex reconstruction. Fig. 14 shows a distribution of $z$-vertex difference between vertices found in the $(x, z)$ and $(y, z)$ planes for events with multiple tracks. The event $z$-vertex is found by taking the average of the $z$ positions determined for all such combinations of tracks. The event vertex in the $x$ and $y$ coordinates is calculated based on the event $z$-vertex and the primary track.

The vertex resolution is estimated by two track MC events of neutrino chargedcurrent interactions. The vertex resolution is degraded mainly due to track mis-reconstruction. Fig. 15 shows the difference between the measured vertex position and the true vertex position in $x, y$, and $z$ for the two track events. The estimated vertex resolution for the two track events is found to be $0.6 \mathrm{~cm}$ in $z$ and $0.4 \mathrm{~cm}$ in $x$ or $y$. 

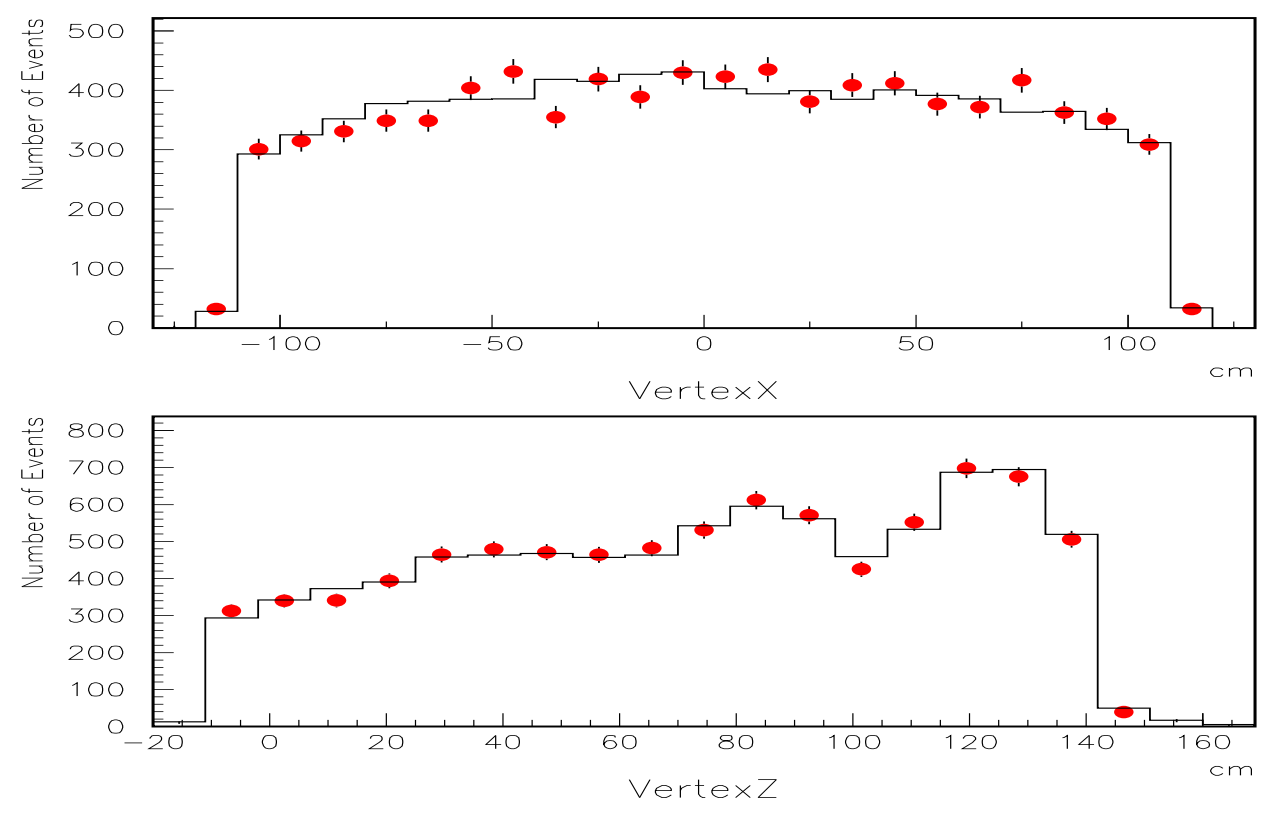

Fig. 13. A comparison of $x$ - and $z$-vertex distributions between data and MC for both single and multiple track events.

\subsection{Track Counting}

An accurate reconstruction of the track multiplicity of an event is crucial for the measurement of neutrino energy spectrum. Charged current quasielastic events have especially simple kinematics which can be used to determine the incoming neutrino energy. These events always have one or two tracks depending on whether the outgoing proton is detected.

The efficiency of finding multiple tracks is studied using the MC CCqe events, and defined to be the fraction of reconstructed events relative to the generated events for a given track multiplicity. Both the reconstructed track and the generated track are required to have three or more hits. The efficiency is estimated to be $93 \pm 2 \%$ and $86 \pm 4 \%$ for single track CCqe events and two track CCqe events, respectively. For single track events, the uncertainty in the track counting efficiency is dominated by the systematic error due to the gain variation of the IIT's, and estimated to be $1 \%$.

For two track events, the sources of the uncertainty in the track counting efficiency are difference in noise between MC and data, tracks due to noise hits around the vertex, and noise hits near the primary track. Due to the difference in noise between data and $\mathrm{MC}$, the track reconstruction may perform differently. 


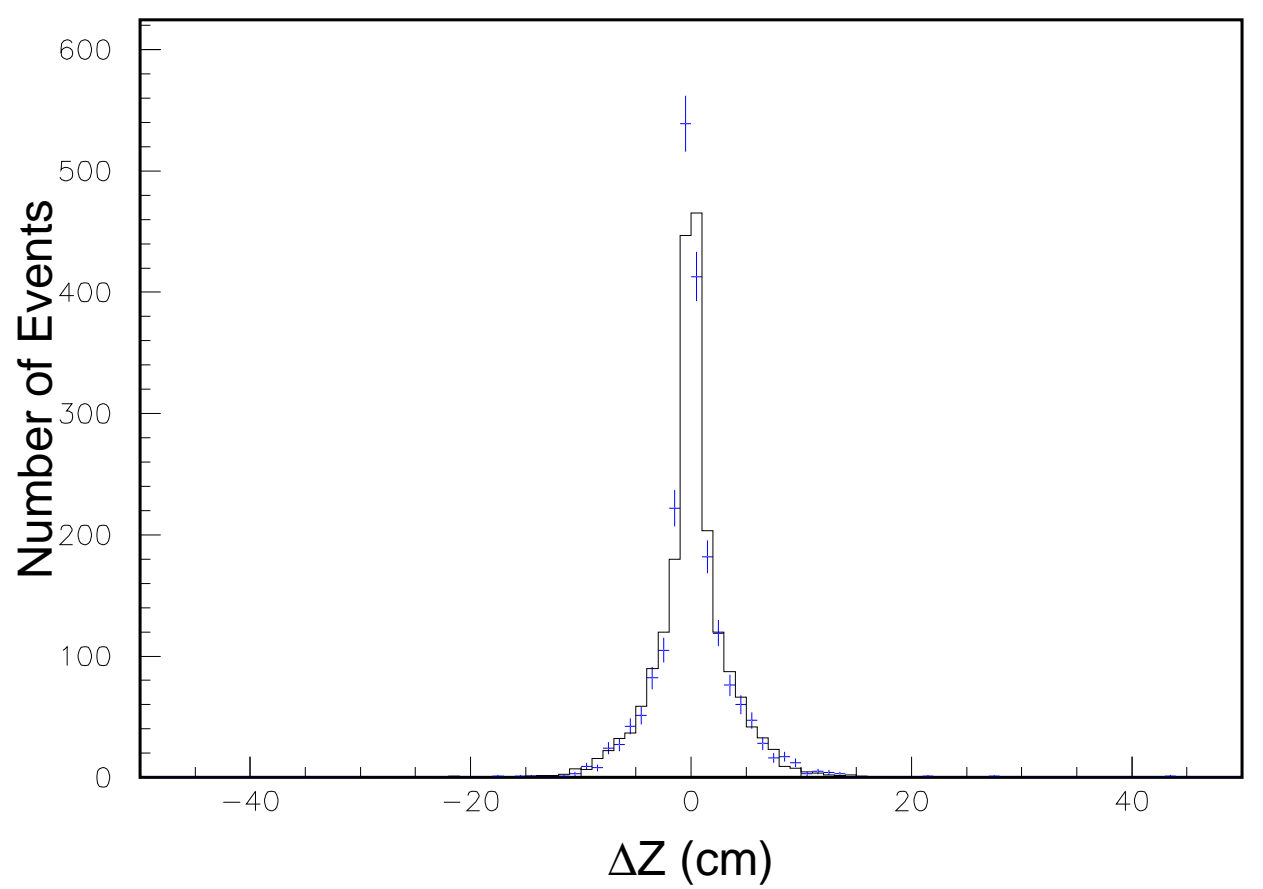

Fig. 14. The $z$-vertex difference between vertices found in the $(x, z)$ and $(y, z)$ planes for two track events. At least three hits are required for both primary track and second track. Points are for data and the histogram is for MC.

In order to estimate the systematic uncertainty due to difference in noise contamination between the data and $\mathrm{MC}$, noise contamination is varied in the $\mathrm{MC}$ by $\pm 50 \%$ from the default value. After the variation, track finding is performed again. We find the track counting efficiency varies by at most $1.8 \%$ with respect to the default noise containmination in the $\mathrm{MC}$ for both single track and two track events.

Single track events can sometimes be reconstructed as two track events because of noise hits around the vertex. The size of this effect is studied using MC generated single track events with three or more hits. The probability is calculated by counting number of reconstructed two track events, and found to be $2.7 \pm 0.1 \%$ for the MC CCqe events.

On the other hand, if there are nearby noise hits along the primary track, single track events might be reconstructed as two track events. This type of nearby noise hits contributes to the uncertainty in track counting for two track events. For single track events, the vertex position is relocated to the middle of the track and the track finding is repeated around the new vertex. The uncertainty in track finding due to accidental noise hits around the primary track is found to be less than $1 \%$. 

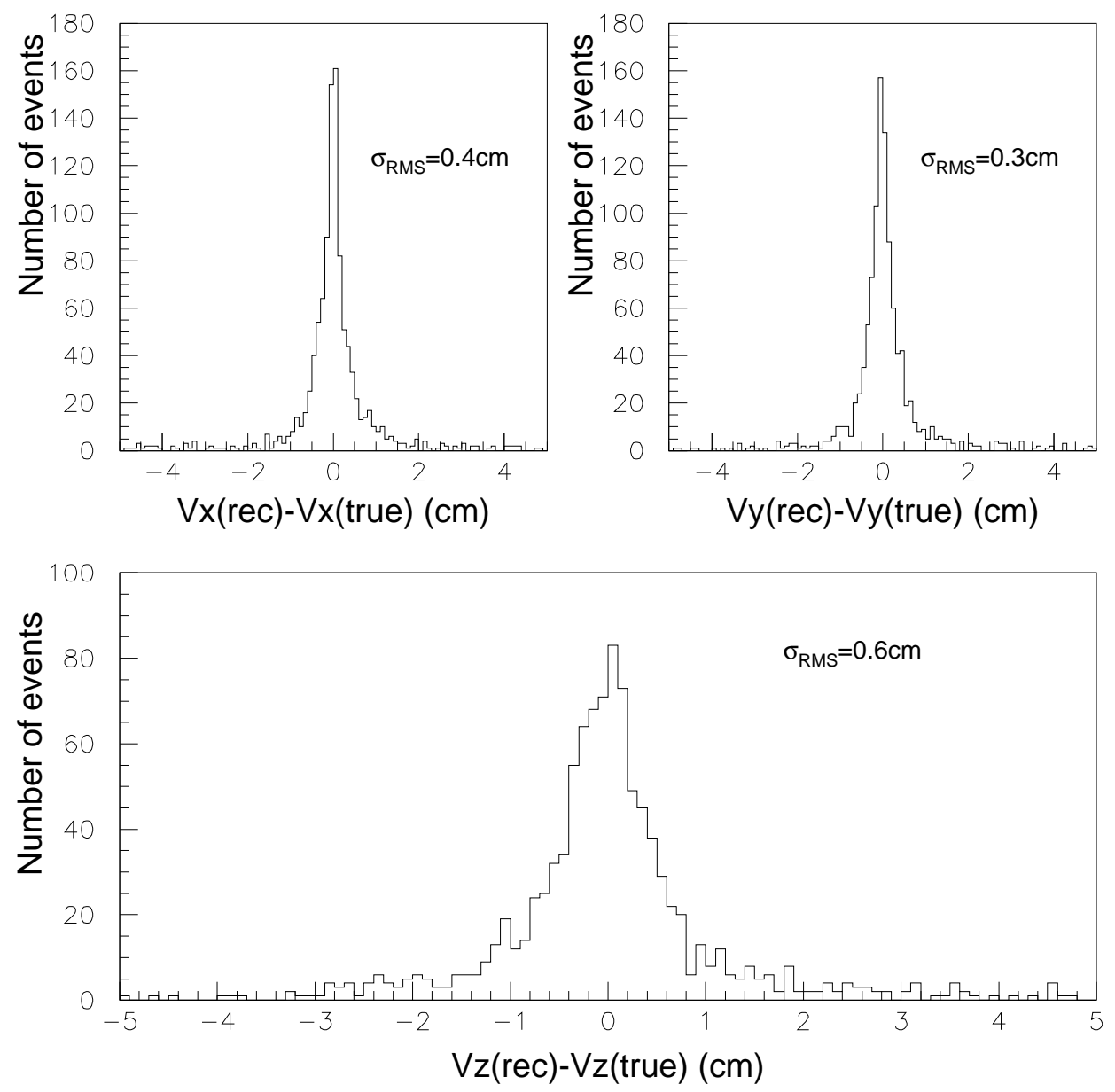

Fig. 15. The vertex resolution of two track charged-current events.

The track counting inefficiency can be explained by loss of tracks or additional fake tracks through reconstruction. The efficiency of finding the second track is calculated as a function of number of hits in the SciFi layers using MC. Events with two generated tracks are reconstructed, and only those with a primary track and a vertex are selected. The second generated track should have at least two hits. The efficiency of finding the second track is defined to be the ratio of number of events with two reconstructed tracks to the number of events with two generated tracks.

Fig. 16 shows the efficiency of finding the second track as a function of number of hits in the SciFi layers. The upper plot is for all types of MC events, and the lower plot is for the CCqe events.

For the second tracks with three or more hits, the inefficiency comes from hit finding inefficiency and limited performance of the track reconstruction as for the primary track. For the second tracks with two hits, the inefficiency is mainly due to noise hits around the vertex or near-by noise hits along the primary track. An average efficiency of finding the second track with three or 

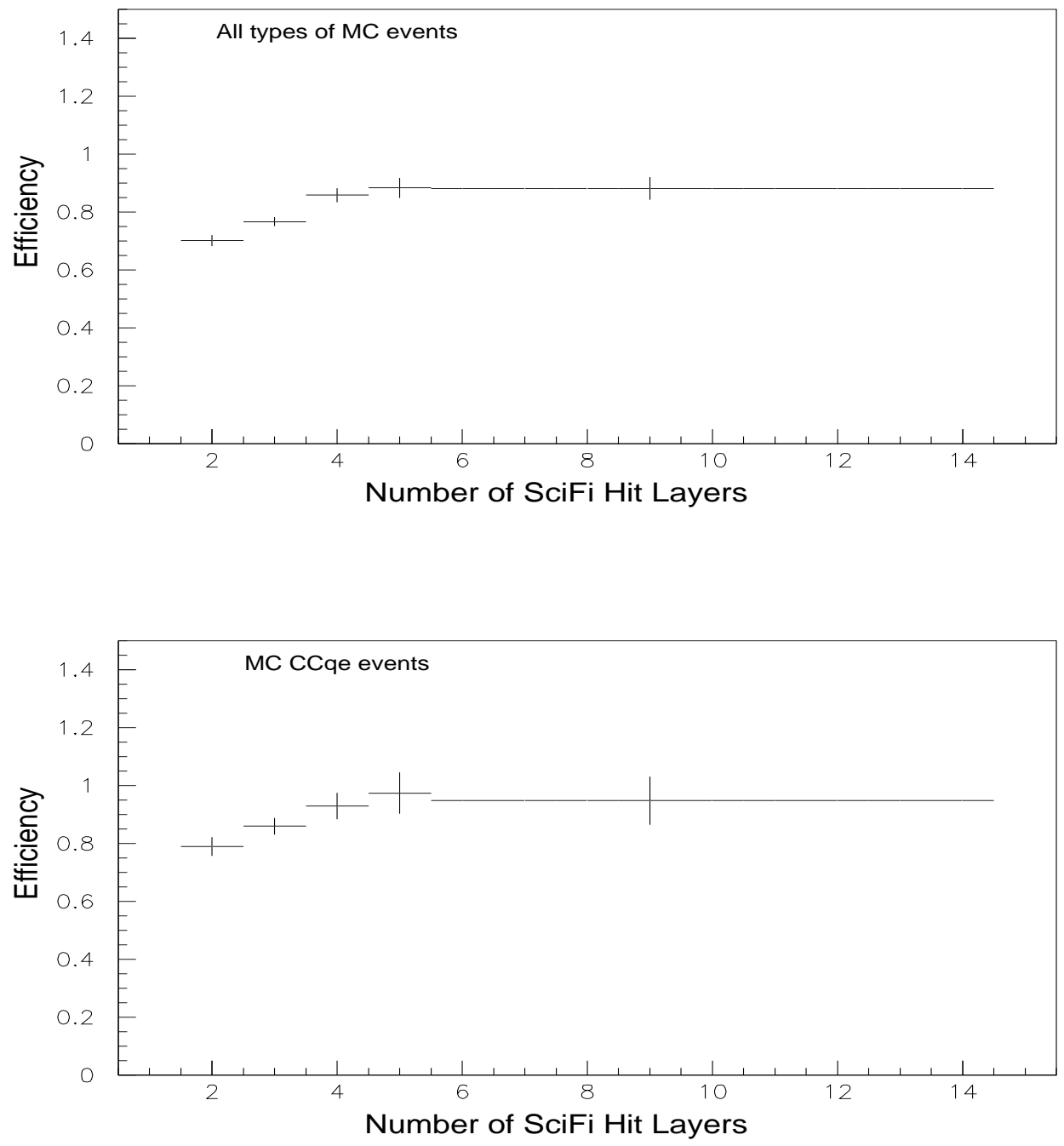

Fig. 16. The efficiency of finding the second track as a function of number of hits in the SciFi layers. The upper plot is for the all events and the lower plot is for the CCqe events.

more hits is found to be $82.1 \pm 0.4 \%$ for all types of $\mathrm{MC}$ events and $90.1 \pm 0.5 \%$ for MC CCqe events.

\section{Event Reconstruction}

In the previous section, we described the reconstruction of tracks and vertices. In this section, we demonstrate their performances using distributions of typical physics variables.

Fig. 17 shows a typical $\nu_{\mu}$ CCqe candidate event. The long track is the muon which leaves hits in the VETO and LG, and ranges out at the MRD. The 


\begin{tabular}{lcc}
\hline \hline Performance Quantity & Data & MC Events \\
\hline Hit finding efficiency $\left(\epsilon_{\text {hit }}\right)$ & $96.5 \pm 0.5 \%$ & $96.8 \pm 0.1 \%$ \\
\hline Primary tack finding efficiency $\left(\epsilon_{t r k}\right)$ & $93.6 \pm 0.8 \%$ & $92.7 \pm 0.5 \%$ \\
\hline Position resolution & $0.64 \pm 0.07 \mathrm{~mm}$ & $0.57 \pm 0.02 \mathrm{~mm}$ \\
\hline Vertex resolution & & \\
Single track & N/A & $6 \mathrm{~mm}$ (Transverse) \\
& N/A & N/A \\
Two track & N/A & $4 \mathrm{~mm}($ Transverse) \\
& & $6 \mathrm{~mm}($ Longitudinal) \\
\hline Tracking counting & & \\
1-track efficiency & N/A & $93 \pm 2 \%$ \\
2-track efficiency & N/A & $86 \pm 4 \%$ \\
\hline \hline Table 1
\end{tabular}

Table 1

Summary of tracking performance of the SciFi. Position resolution is measured using the neutrino data. Vertex resolution is estimated using MC events. Track counting efficiency is calculated for the CCqe events. Errors on the hit efficiency, tracking efficiency, and track counting efficiency are the quadratic sums of statistical and systematic errors.

short track is most likely a proton.

Most of the protons from neutrino interactions have relatively low momenta and thus make short second-tracks in the SciFi. Fig. 18 shows the track length distribution for the second tracks in the 2-track sample. Both Monte Carlo prediction and data agree well, demonstrating the validity of the neutrino event generation and the tracking method.

\section{Conclusions}

The K2K long-baseline neutrino experiment has taken data for three years. The SciFi is used to identify charged particle tracks in the K2K near detector.

The SciFi hits are calibrated using cosmic ray data and electro-luminescent calibration data. MC simulation reproduces the distribution of the number of hit pixels in the cosmic ray data very well. The stability of the SciFi is checked periodically by obtaining the distribution of hit efficiency and calculating the mean of the hit pixel distribution. The stability is better than $5 \%$ and $1.2 \%$ in the mean of pixel distribution and the hit efficiency, respectively. 


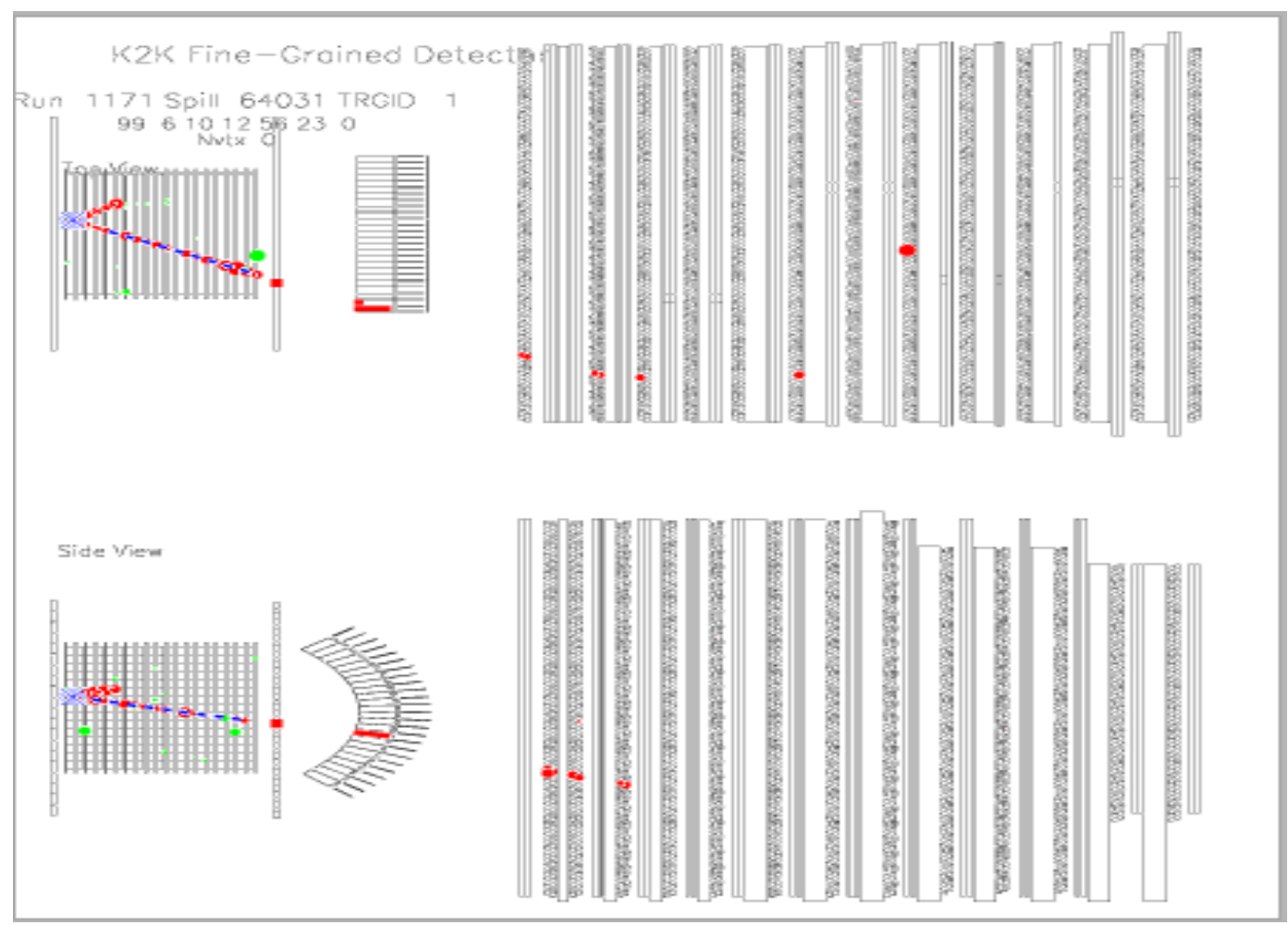

Fig. 17. A candidate $\nu_{\mu}$ CCqe event. The long track is the muon and the short track is most likely a proton.

A track reconstruction algorithm, optimized for charged current neutrino interactions, is employed to find tracks and event vertices. The track finding efficiency is monitored and found to be stable throughout the data-taking period. Using the estimated vertex resolution, the vertex reconstruction alogirthm is found to be good enough for defining the event fiducial volume with an accuracy better than 1\%. A track fit using a Kalman filtering technique is used for the best estimation of hit resolution, and found to improve the fiber alignment.

Accurate track counting is crucial for the measurement of neutrino energy spectrum and in achieving the physics goals of the $\mathrm{K} 2 \mathrm{~K}$ experiment. The track counting uncertainty is found to be at $2 \%$ and $4 \%$ level for the single track and two track events, respectively. Overall tracking performance of the SciFi is summarized in Table. 1.

The performance of the SciFi tracker is well demonstrated by physics variables of neutrino interaction. The distributions of variables for muon track and proton track show a good agreement between data and MC events. 


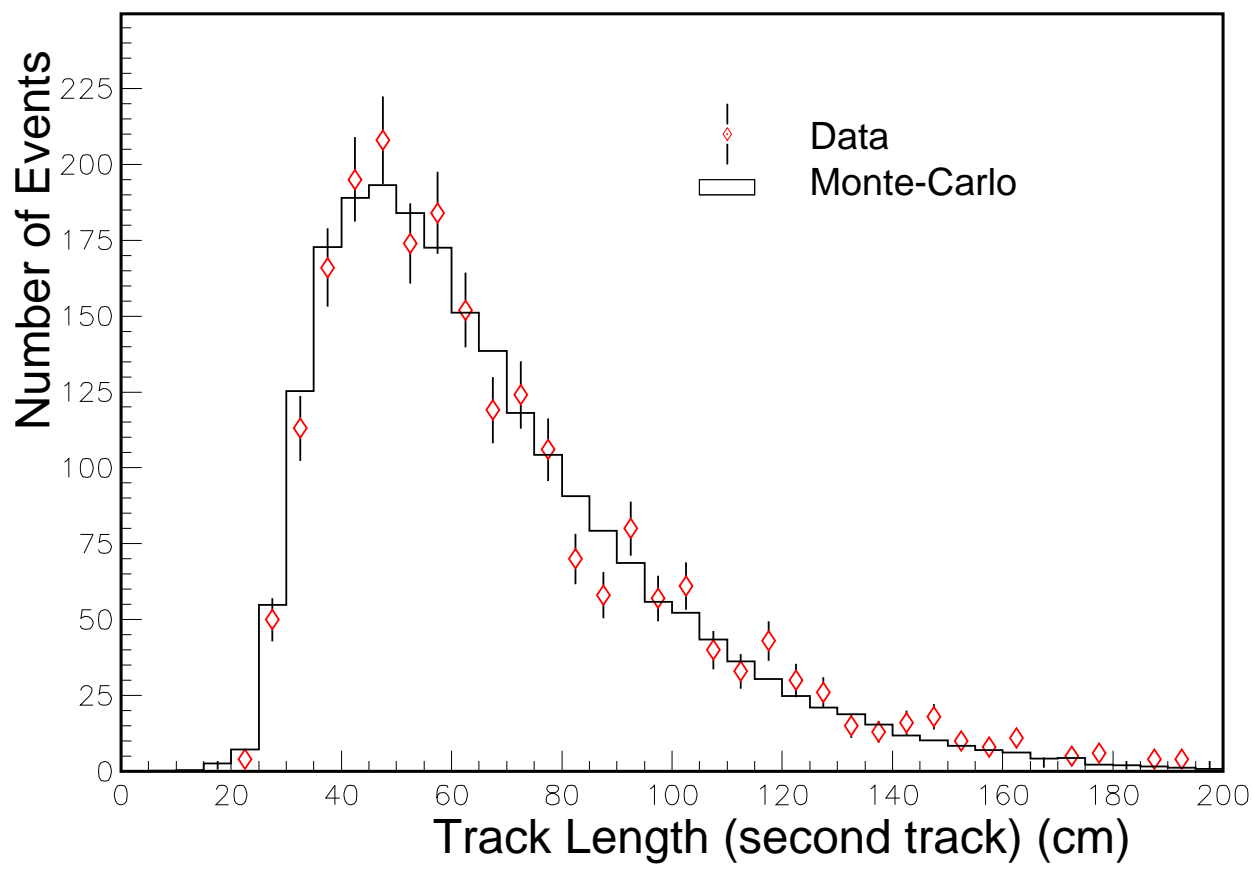

Fig. 18. A comparion of track length for the proton between neutrino data and MC events in two track sample.

\section{Acknowledgements}

The vital contributions of the KEK staff and the technical staffs of the participating institutions are gratefully acknowledged. This work is supported by the Ministry of Education, Science and Culture of Japan, the U.S. Department of Energy, the National Science Foundation of the U.S., the Korea Research Foundation, the Korea Science and Engineering Foundation, and the CHEP in Korea.

\section{References}

[1] Y. Fukuda et al., Super-Kamiokande Collaboration, Phys. Rev. Lett. 81 (1998) 1158; M. Nakahata et al., Super-Kamiokande Collaboration, Nucl. Instr. and Meth. A 421 (1999) 113.

[2] S. H. Ahn et al., K2K Collaboration, Phys. Lett. B 511 (2001) 178; K. Nishikawa et al., KEK-PS proposal (E362) (1995).

[3] T. Ishii et al., K2K Collaboration, Nucl. Instr. and Meth. A 482 (2002) 244.

[4] R.E.Ansorge et al., UA2 Collaboration, Nucl. Instr. and Meth. A 265 (1988) 33; P. Annis et al., CHORUS Collaboration, Nucl. Instr. and Meth. A 412 (1998) 19. 
[5] A. Suzuki et al., K2K Collaboration, Nucl. Instr. and Meth. A 453 (2000) 165.

[6] R. Brun et al., GEANT3, CERN DD//EE/84-1, revised 1987.

[7] R.E. Kalman, Trans. ASME, J. Bas. Eng. 82D (1960) 35 ; and R.E. Kalman and R.S. Bucy, Trans. ASME, J. Bas. Eng. 83D (1961) 95.

[8] P. Billoir, Nucl. Instr. and Meth. 225 (1984) 352.

[9] R. Fruhwirth, Nucl. Instr. and Meth. A262 (1987) 444.

[10] V. Innocente and E. Nagy, Nucl. Instr. and Meth. A324 (1993) 297; E. J. Wolin and L. L. Ho, Nucl. Instr. and Meth. A329 (1993) 493; and R. Harr, IEEE Trans. on Nucl. Sci. V42 (1995) 134. 